\title{
Current Dental Adhesives Systems. A Narrative Review
}

\author{
Egle Milia*, Enzo Cumbo**, Rielson Josè A. Cardoso*** and Giuseppe Gallina**
}

*Dept of Conservative Dentistry, University of Sassari, Sassari, Italy; **Section of Oral Sciences, Dept. Surgical and Oncological Disciplines, University of Palermo, Palermo, Italy; ***College of Dentistry and Center of Research São Leopoldo Mandic, Campinas, Brazil

\begin{abstract}
Adhesive dentistry is based on the development of materials which establish an effective bond with the tooth tissues. In this context, adhesive systems have attracted considerable research interest in recent years. Successful adhesive bonding depends on the chemistry of the adhesive, on appropriate clinical handling of the material as well as on the knowledge of the morphological changes caused on dental tissue by different bonding procedures.

This paper outlines the status of contemporary adhesive systems, with particular emphasis on chemical characteristics and mode of interaction of the adhesives with enamel and dentinal tissues.

Dental adhesives are used for several clinical applications and they can be classified based on the clinical regimen in "etch-and-rinse adhesives" and "self-etch adhesives". Other important considerations concern the different anatomical characteristics of enamel and dentine which are involved in the bonding procedures that have also implications for the technique used as well as for the quality of the bond. Etch-and-rinse adhesive systems generally perform better on enamel than self-etching systems which may be more suitable for bonding to dentine.

In order to avoid a possible loss of the restoration, secondary caries or pulp damage due to bacteria penetration or due to cytotoxicity effects of eluted adhesive components, careful consideration of several factors is essential in selecting the suitable bonding procedure and adhesive system for the individual patient situation .
\end{abstract}

Keywords: Dental adhesive, enamel, dentine, composite resin, phosphoric acid.

\section{INTRODUCTION}

Nowadays, there are several tooth-colored dental restorative materials available in the market such as glass-ionomers, resin modified cements, compomers and composites.

\section{Glass-Ionomer Cements, Resin-Modified Glass-Ionomer Ce- ments and Polyacid-Modified Composites}

Glass-ionomer cements are fluoride-containing silicate glass and polyalkenoic acids based which are set by an acid-base reaction between all the components.

Fluoride is one of the ionic constituents that are released from the glass during the setting reaction; during the first $24 \mathrm{~h}$ an initial high release from glass-ionomers is likely due to the burst of fluoride released from the glass particles when reacting with the polyalkenoate acid during the setting reaction.

There is a difference between conventional glass-ionomer cements and metal-reinforced glass-ionomers which seem to release less fluoride because of the initial lower fluoride content of these materials due to the replacement by silver or the formation of silver fluoride which binds the fluoride ions into the cement preventing leaching of the fluoride.

After the initial burst, the high amounts of fluoride rapidly decrease after $24-72 \mathrm{~h}$ and plateaued to a nearly constant level within about 20 days so a prolonged long-term fluoride release occurs when the glass dissolves in the acidified water of the hydrogel matrix.

The amount of fluoride in saliva measured immediately after placement glassionomer restorations is approximately from 0.04 to 0.8-1.2 ppm.

*Address correspondence to this author at the Dept of Conservative Dentistry, University of Sassari, Sassari, Italy; Tel: +39-079228437;

E-mail:emilia@uniss.it

\section{Resin-modified Glass-ionomer Cements and Polyacid-modified Composites}

To overcome the problems of moisture sensitivity and low initial mechanical strengths, typical for conventional glass-ionomers, resin-modified glass-ionomer cements and polyacid-modified composites have been developed.

Polyacid-modified resin composites (compomers) claim to combine the mechanical and aesthetic properties of composites with the fluoride-releasing advantages of conventional glass-ionomer cements so the resultant material is a low-fluoride-releasing resin composite that contain vinyl groups that can be polymerized by visible light activated initiators [1].

Fluoride is able to diffuse out of the composite after that the fluids, from the oral cavity, diffuse into the resin and swell it and open up the polymer networks even if the fluoride release from compomers is lower compared with conventional glass ionomers or resin-modified glass ionomers.

Resin-modified glass-ionomers were basically formed by adding methacrylate components to the polyacrylic acid; polyacidmodified resin composites consist of conventional macromonomers also used in composites, such as bisphenol-glycidyl-dimethacrylateor urethane-dimethacrylate, together with small amounts of acidfunctional monomers.

The filler glass is identical to the ion-leachable glass fillers used in conventional glass-ionomer cements: silicate-based glasses and sodium fluoride, but in smaller sizes as known from composites.

Initial setting is performed by light-activated polymerization which is followed by an acid-base reaction that arises from sorption of water. In fact these materials absorb water from the oral cavity after a period of time, so some expansion may make up for the early polymerization shrinkage however, the adhesive layer limits the uptake of the fluoride into surrounding tooth structure. 
The fluoride release and mechanical properties of compomers vary considerably between materials and unfortunately some mechanical properties are inferior to traditional composites; improvements in compomer formulations by reducing the filler size of 0.8 microns have improved their handling, polishability, and stain resistance.

Compomers have to be used like composites and the acid etching step is an absolute necessity to improve the retention rate and decrease marginal leakage $[2,3,4]$.

As a result, compomers must be placed exactly like traditional resin composites and same clinicians prefer compomers only in Class V, especially if enamel is available for bonding and a limited fluoride release is desirable because those materials are indicated for other esthetic clinical situations where they will not be subjected to significant occlusal loads.

Flowable compomers have been used clinically on a limited basis similar to flowable composites in proximal boxes for Class II restorations demonstrating the least amount of overall leakage under its packable composite counterpart, compared with other flowable composite combinations.

Recently, a new category of hybrid material called giomers are available, that include pre-reacted glass-ionomers to form a stable phase of glass-ionomer fillers and unlike compomers, fluoroalumino-silicate glass particles react with polyacrylic acid prior to inclusion into the resin matrix.

For giomers, no initial 'burst' effect seem to be present and amounts of fluoride leached from those restorative materials is slightly higher than from composites and compomers but lower compared to glass-ionomers.

\section{Composite}

Composite resin is a multiphase material that exhibits the properties of both phases where the phases are complimentary, resulting in a material with enhanced properties [5].

The first composite formulation, introduced in 1870s, was based on alumino-fluro-silicate glasses and phosphoric acid and specifically the matrix phase was the aluminum phosphate salt formed from the partial acid dissolution of the glass particles instead the dispersed phase was residual glass particles.

Restorations made using these material were brittle, required mechanical retention, and had a short average longevity.

The first polymeric tooth-colored composite used in dentistry was developed in the 1940s and it was based on methylmethacrylate and consisted of a polymethylmethacrylate powder, methyl methacrylate monomer, benzoyl peroxide, and n,n-dimethlyparatoluidine.

Using a redox initiator combination of benzoyl peroxide and $\mathrm{n}$,ndimethlyparatoluidine the polymerization was initiated at room temperature moreover these materials had several problems in fact they were initially aesthetic but they showed poor colour stability, high polymerization shrinkage, a lack of bonding to tooth structure, and high coefficient of thermal expansion.

Silica fillers were introduced in the 1950s and these composites had improved mechanical properties and good aesthetics but unfortunately, during the polimerization reaction, a significant shrinkage was present and also they did not bond to enamel and dentine and there was no significant bonding between the silica particles and the polymer matrix; as a consequence they didn't have a good resistance.

A coupling agent such as g-methacryloxpropyl-trimethoxy silane or vinyl triethoxysilane was introduced in order to bond the particles to the matrix and the resulting new resin had improved mechanical properties and wear resistance but both problems polymerization shrinkage and lack of bonding to tooth structure were still unsolved.
Thanks to Bowen in 1962 a new formula was introduced on the market called Bis-GMA or Bowen's resin that had high viscosity so only a small quantity of filler particles could be incorporated in fact a new matrix was produced incorporating triethylene glycol dimethacrylate (TEGDMA) as a diluent to reduce the viscosity.

During the following years a lot of composite formulations have been experimented using various diluents monomers such as methyl methacrylate (MMA) and ethylene glycol dimethacrylate (EGDMA), and an additional high molecular weight monomer based on a urethane dimethacrylate (UDMA).

All these monomers contain two reactive double bonds that are capable to form, when polymerized, covalent bonds between the polymer chains (cross-links). Cross-linking improved the properties of the matrix phase, and the composite produced had improved mechanical and physical properties.

Composite classification:

Composite restoratives can be classified using several parameters but one of the most used classification system is based upon filler particle size and in order to improve mechanical and esthetics properties fillers formulations have been changed a lot of times $[7,8]$.

Nowadays the used classification divides composite resins as follows

- microfills,

- hybrids,

- packables,

\section{Microfilled Composites}

They were developed to provide the dental profession with a material that possessed outstanding polishability and aesthetics and for that purpose particles ranging from $0.04 \mathrm{um}$ to $0.4 \mathrm{um}$ were incorporated.

Because of the high surface area-to-volume ratio of the filler, the first versions of those resins, which contained 35 to 65 weight $\%$ and 20 to 60 volume $\%$ glass particles, had a limited quantity of fillers that caused a large viscosity increases.

Further studies led to another way to increase the volume of small particles and that was the use of pre-polymerized particles so submicron-sized particles were mixed with Bis-GMA and TEGDMA monomers at elevated temperatures.

That mixture obtained was cured at elevated temperature and pressure, using benzoyl peroxide as an initiator and let chill, the following step was to ground the resin in order to obtain particles having a size range of 1 to $200 \mathrm{~mm}$ that allowed higher filler loadings. However, the pre polymerized particles cannot be bonded to the matrix phase using silane coupling agents, in fact, interfacial bonding requires diffusion of the matrix monomers into the particles, with subsequent polymerization to provide micromechanical interlocks.

In any case the presence of pre polymerized particles allows the filler content to be maximized and polymerization shrinkage to be minimized and as a result micro filled composites have a lower elastic modulus and lower fracture strength than materials that contain higher concentrations of filler.

Another subclass of micro fills that has recently been produced is referred to as flowable that are composed of traditional composite resins with a filler loading reduced to 30 to 55 volume $\%$, compared with 57 to 72 volume \% for traditional hybrid resin composites.

In the market different type of flowable composites can be found and their fluidity may be very different between various products so they are recommended for use in low stress areas such as very small Class I restorations similar to pit and fissure sealants or conservative composite restorations and where limited access to 
the restoration made it difficult to adapt other composite types to the walls of the preparation.

\section{Hybrid Composites}

Nowadays the most used composites are generally called hybrid; they can be divided in hybrids, micro, and nano-hybrids.

The "hybrid" contains submicron inorganic filler particles (.04 $\mathrm{mm})$ and small particles $(1 \mathrm{~mm}-4 \mathrm{~mm})$. That combination of various sizes of filler particles improves physical properties and allows an acceptable levels of polishability.

Another subcategory of hybrid composites called micro-hybrid contains both submicron particles $(0.04 \mathrm{~mm})$ and small particles $(0.1 \mathrm{~mm}-1.0 \mathrm{~mm})$ that allows for a finer polish, along with improved handling.

The latest version of micro filled hybrids that uses nano filler technology, contains nano meter-sized filler particles $(.005-.01 \mathrm{mi}-$ crons) throughout the resin matrix, in combination with a more conventional type filler technology.

Nanohybrids may be classified as the first truly universal composite resin with handling properties and polishability of a micro filled composite, and the strength and wear resistance of a traditional hybrid. Tanks to the smaller particle size these resins can be used in any situation similar to the microhybrids, with possibly a slight improvement in polishability

\section{Packable Composites}

In order to increase the use of composites by older dentists who are used to using amalgams, packable composites were introduced in the market that have a higher viscosity and are less sticky even if their viscosity depends on the temperature. In fact when initially placed, these materials were more viscous than traditional hybrid composites; however, after placement the viscosity decreased as the temperature of the material equilibrated with the temperature of the oral cavity $[9,10]$.

The viscosity increase is obtained through changes in the particle size distribution and incorporation of fibers but they do not have substantially better mechanical properties than hybrid composites consequently they would not be expected to perform better clinically actually they have shown decreased wear resistance. As a conclusion their use as a direct dental restorative may be limited.

Nowadays the entire restorative dentistry is based on the use of composites and adhesives that allows to create a strong bond between dental tissue (enamel and dentin) and restorative material used.

Modern dental adhesive systems are used to improve the bond between composite resin materials and the cavity walls of the prepared tooth. The adhesion of the composite resin to the adhesive is achieved by a process of co-polymerization of residual double bonds (-C=C-) in the oxygen inhibition layer of the adhesive. Bonding to the tooth substrates is based upon an exchange process by which inorganic tooth material is exchanged for synthetic resin. Demineralization by phosphoric acid $[11,12]$ or acidic monomers is a prerequisite to remove the inorganic component by which microporosities are exposed on the enamel and dentine tooth surface. The subsequent infiltration and in situ polymerization of the monomers allow for the formation of the so called hybrid layer [13,14] (Fig. 1). This process, is primarily based on diffusion with micro-mechanical interlocking, which is considered as the gold standard in terms of bonding effectiveness. Furthermore, lately attention has been given to additional chemical interaction between functional monomers and tooth substrate components which potentially play a critical role in the overall adhesion process $[15,16]$.

Other important considerations concern the different anatomical characteristics of enamel and dentine which are involved in the bonding procedures. Morphologically, the dentine matrix is com-

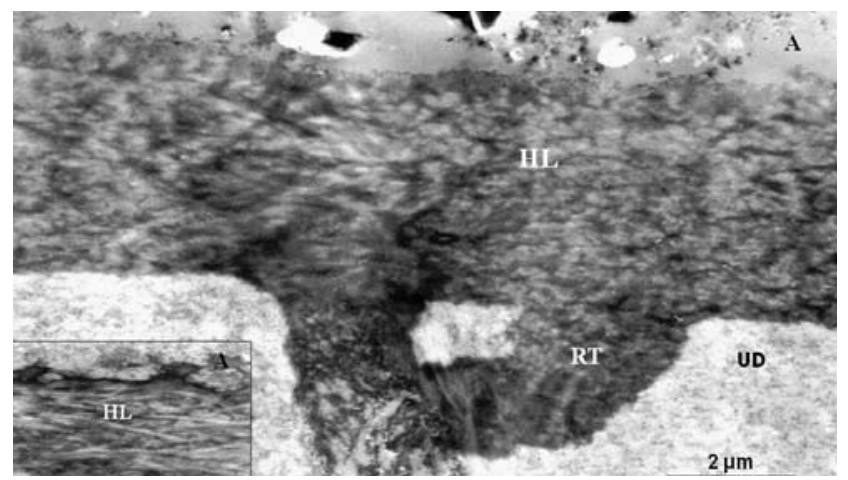

Fig. (1). Transmission electron microscopy (TEM) image of the thick hybrid layer and resin tags formed by OptiBond Solo (Kerr Corporation, Orange, CA, USA), a sigle bottle, etch-and-rinse adhesive system. The demineralized and stained sections in ( $\mathbf{a}$ and $\mathbf{b}$ ) show the dense micro-infiltration of the resin within the fully demineralized collagen fibrils and tubule apertures. A resin-injected lateral branch interconnects tubules.

$\mathrm{HL}=$ hybrid layer; $\mathrm{RT}=$ resin tag; $\mathrm{A}=$ adhesive $; \mathrm{UD}=$ unaltered dentine

posed of tubules that run continuously from the dentine-enamel junction to the pulp, filled with intra-tubular fluids and surrounded by a hypermineralized peritubular dentine, and a softer intertubular matrix of mineralized collagen fibrils. These peculiar features make the dentine a highly hydrophilic semi-permeable barrier between enamel and pulp explaining the diverse response to the bonding procedure compared to that of enamel $[17,18,19,20]$. Moreover, such features may hinder effective bonding to dentine and this may consecutively lead to pulp damage due to eluted cytotoxic adhesive components or due to bacteria, which have penetrated the gap between the restoration and the cavity wall [21]

Thus, knowledge of the adhesive ingredients is instrumental to better understanding the efficiency and effect of the adhesive bonding.

This paper outlines the status of contemporary adhesive systems, with particular emphasis on the chemistry of adhesives and the different mechanisms of bonding to enamel and dentine.

\section{CHEMICAL CHARACTERISTICS OF THE ADHESIVE SYSTEMS}

Irrespective of the bonding mechanism, the basic ingredients of the adhesive systems are acrylic resin monomers, organic solvents, initiators, inhibitors, and sometimes filler particles [22]. Nevertheless the proportional composition and the chemistry of these ingredients differ between the different classes of adhesives.

\section{Self-etch Systems}

Resin monomers are the key components of the adhesive formula and include cross-linkers and functional monomers. Three distinct parts characterise monomer structures: i) one or more polymerizable groups linked to ii) a spacer, and iii) a functional group (Fig. 2). Polymerizable groups are methacrylate and acrylates. Being part of a large molecule (e.g. Bis-GMA) they generally show a hydrophobic behavior. Methacrylates are less reactive and sensitive to oxygen inhibition than acrylates and may therefore be less cytotoxic [23]

The main function of the monomer spacer is to keep both functional and polymerizable groups well separated. In addition, the spacer influences the properties of the monomer and the resulting polymer. Design and size of the spacer group influences hydrophilicity of the resulting polymer [24]. The size of the spacer determines moreover the viscosity of the monomers and the related capacity of wetting and infiltration of the monomers. Voluminous 


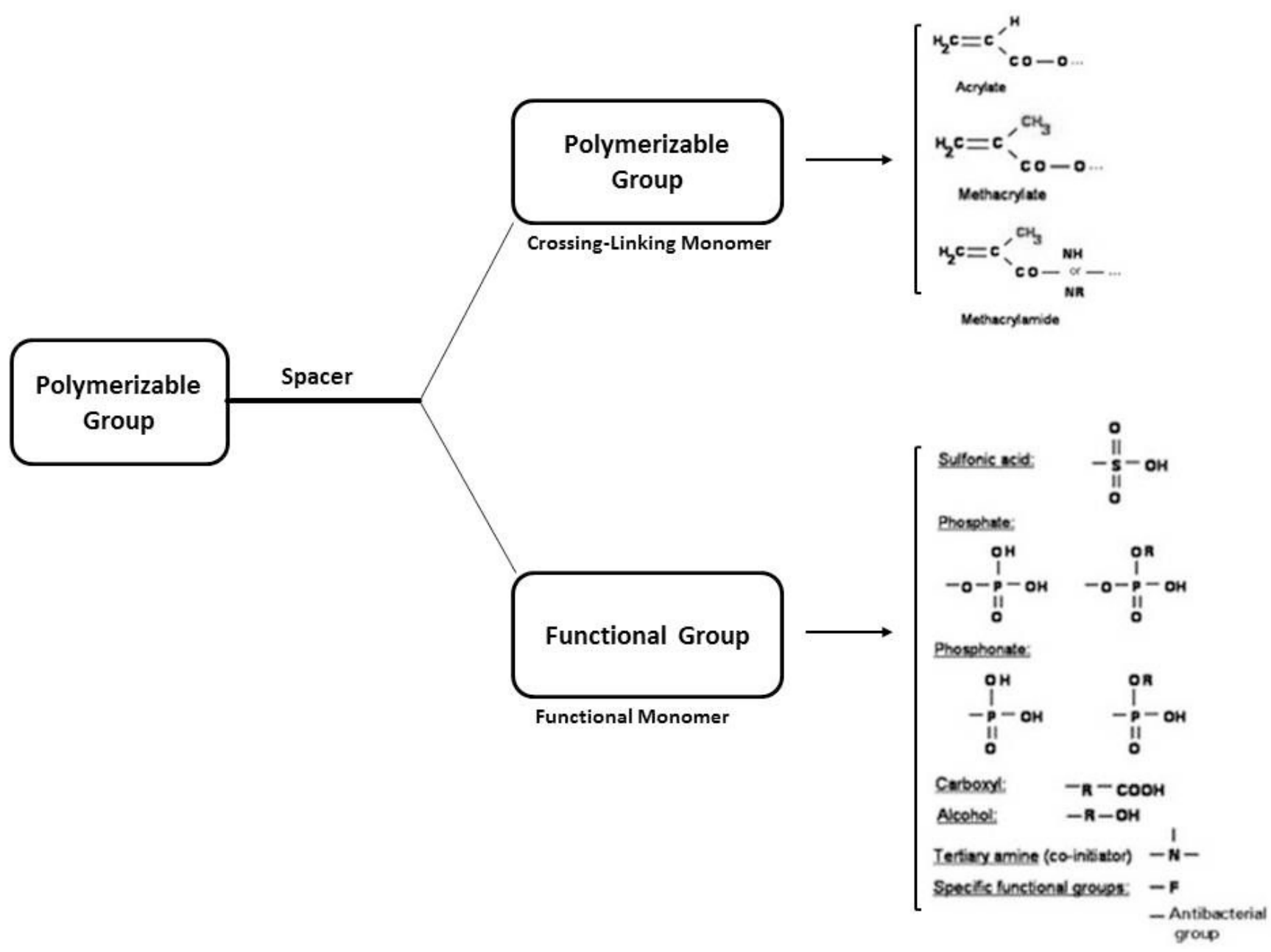

Fig. (2). Make-up of either a cross-linking or a functional monomer commonly used in adhesive dentistry. It also includes the structure of adhesive methacrylates monomers (Van Landuyt et al. 2007).

groups may cause other monomers not to reach the polymerizable group, thereby compromizing good polymerization (steric hindering) [22].

Furthermore, the polarity of the spacer may have an influence on the solubility of the monomer in water and other solvents: for instance, the hydrophilicity of the spacer may cause water uptake leading to higher hydrolysis susceptibility of the monomers, as well as hygroscopic expansion of the polymer $[23,24,25]$.

The functional group among functional monomers has characteristically hydrophilic properties. This group may serve for several purposes, such as wetting and demineralization of the tissue, release of fluorides, and antibacterial effects. Moreover, the hydrophilic functionality acts as adhesion promoter enhancing the bond strength of adhesives to dentine substrate [26]. The most common functional groups used in commercial monomers are phosphate, carboxylic acid and alcohol groups (Fig. 2) Apart from 'adhesion-promoting' or wetting effects, these proton-releasing functional groups may establish surface demineralization to a certain extent when applied in a sufficient concentration. A ranking on etching aggressiveness can be made according to the acidity of these groups [27]. Occasionally, particular functional groups are added to a monomer to enhance demineralization capacity, allow for antibacterial properties [28,29,30,31] and desensitizing effects [32], or to increase the biocompatibility of the monomers. The conversion rate of the monomers is an important determinant of the physico-mechanical strength of the resulting polymer. Unfortunately, the conversion rateis not $100 \%$ for resin based composites and for simplified adhesives $[33,34,35,36]$. This poor conversion rate may result in high permeability, water sorption, nanoleakage, degradation of the toothcomposite bond, leaching of residual uncured monomers and thus low biocompatibility of dental adhesives [23]. For this reason, the biocompatibility of resin monomers has intensively been investigated $[37,38,39]$.

\section{MECHANISMS OF ADHESIVE BONDING TO ENAMEL AND DENTINAL TISSUES}

Historically, dental adhesives were classified in "generations", which - after some years - became rather confusing. Also a component-based nomenclature did not prove to be practical. Therefore, contemporary adhesives are classified based on the clinical regimen in "etch-and-rinse adhesives" and "self-etch adhesives" [40]..

\section{-Etch-and-rinse Adhesives}

Etch-and-rinse adhesives are often considered as gold standard for predictable adhesion to the tooth. In the three step etch-and rinse adhesives, the first step is the application of the conditioner (mianly phosphoric acid), which is followed by the primer or adhesion promoting agent application (second step), and (third step) by the bonding agent (also named: adhesive resin). The simplified twostep version combines the second and third step but still follows a separate "etch-and-rinse" phase. 
The good enamel bonding of the etch-and-rinse adhesives is attributed to the particular morphology of the interface obtained after etching enamel with phosphoric acid at concentrations in the range of $34-37 \%$ [41,42].

Phosphoric acid treatment of enamel removes the smear layer and typically creates a honeycomb porosity surface. This can be penetrated by a low-viscosity bonding agent absorbed by capillary attraction [43]. After polymerization of the bonding agent, a durable attachment to the enamel surface is achieved by micromechanical interlocking. The predominant mechanism of bonding resin composites to the phosphoric acid-etched enamel is the formation of macro tag-like resin extensions within the space surrounding the enamel prisms and "micro"- tag-like resin extensions resulting from resin infiltration within the tiny etch-pits at the cores of the etched enamel prisms (Fig. 3).

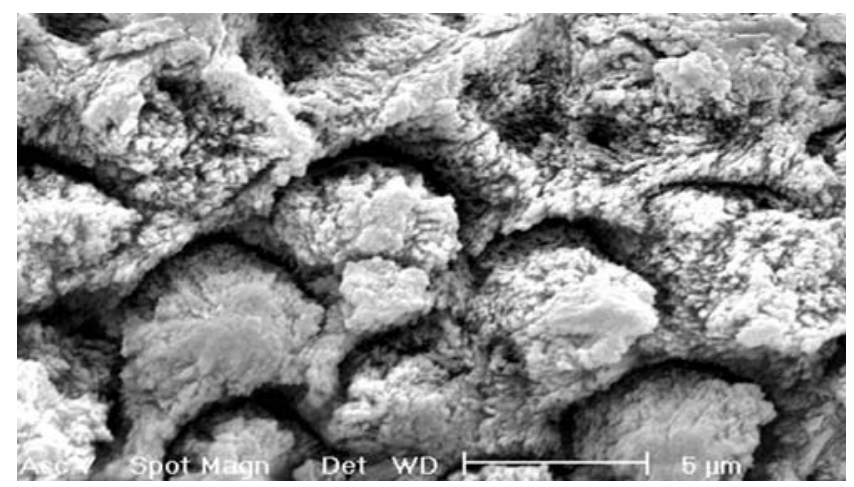

Fig. (3). Scanning electron microscopy (SEM) image of acid etched enamel surface showing dissolution of prism cores with residual micro-pits or micro-craters, and interprismatic enamel border in a geometrical hexagonal ring (honeycomb appearance).

Courtesy of Prof Stefano Eramo, University of Perugia, Italy.

The etching of dentine removes the smear layer, it demineralizes the peritubular dentine and produces a collagen network on the surface and along the tubular surfaces. The hydroxyapatite mineral phase is removed up to $5-8 \mu \mathrm{m}$ from the tissue surface, leaving a surface of exposed collagen fibrils that is nearly totally deprived of hydroxyapatite. This allows for the formation of a very permeable layer permitting a diffusion-based infiltration of hydrophilic adhesives into and around the collagen fibrils and resin tags in the tubules (Fig. 4). Infiltration of demineralized matrix should be as complete as possible to form a hybridized biocomposite of collagen fibrils reinforced by resin known as hybrid layer. Because of the hydrophilic nature of the collagen (and the hydrophobic nature of composite resin materials), different groups of monomers, one with more hydrophilic properties (e.g. HEMA or TEGDMA) and the other with more hydrophobic properties (e.g. Bis-GMA or UDMA) were proposed to be used consecutively. The hydrophilic functional monomers, traditionally contained in the primer, allow for the permeation of the monomer within the demineralized matrix, whereas the hydrophobic monomers contained in the bonding agent, applied later, facilitate adhesion of the composite restorative material to the conditioned dentin surface thus rendered hydrophobic [44].

However, studies involving scanning electron microscopy (SEM) and transmission electron microscopy (TEM) indicated that hybrid layers created by etch-and-rinse adhesives might be poorly infiltrated with resin and it was concluded that the collapse of collagen during the drying process after rinsing the etchant was a main reason for this deficiency $[45,46,47,48,49,50]$. The incomplete infiltration of the collagen network by the monomers has been morphologically observed as voids or microporosity underneath and within the hybrid layer detectable through percolation of silver nitrate as

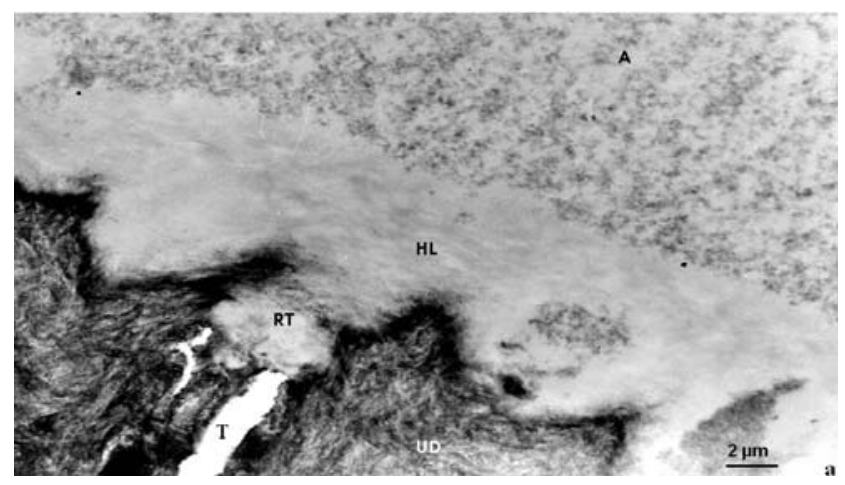

Fig. (4). Non-demineralized, unstained TEM image of the hybrid layer and resin tags formed by the etch-and-rinse adhesive OptiBond Solo (Kerr Corporation, Orange, CA, USA). The thick totally demineralized collagen is reinforced by resin forming an hybrid structure which is no longer dentine nor resin but a sort of tissue engineering. In the case of the etch-and-rinse systems, the hybrid layer is primarily based on a diffusion process with micro-mechanical interlocking of the biomaterial into the demineralized network of collagen.

$\mathrm{HL}=$ hybrid layer; $\mathrm{RT}=$ resin tag; $\mathrm{A}=$ adhesive $; \mathrm{UD}=$ unaltered dentine

nanoleakages [51]. Nanoleakage is considered as a pathway for hydrolytic and enzymatic degradation of the hybrid layer $[52,53,54]$.

Clinicians may believe that the dentinal matrix is a relatively inert, stable collagen scaffold that does not interact with solvated monomers. However, permeation may bring the monomers into close contact with the matrix at the molecular level allowing for potential interactions between the binding sites of the collagen and the monomers [55].

Thus, two other approaches were proposed to study adhesive interactions with demineralized dentinal matrix: (i) analysis of solubility parameter compatibility between the adhesive and collagen substrate and (ii) direct/indirect computer modeling of collagen-ligand interactions.

The use of solubility parameters makes it possible to rank the ability of chemicals to re-expand the collapsed collagen network by breaking collagen inter-peptide hydrogen-bonds which maintain collagen in a stiffened and collapsed state [56]. Such interactions are studied by the application of Hoy's solubility parameters [57]. Hoy's solubility parameters provide estimates of the relative contribution of dispersion forces $(\delta \mathrm{d})$, polar forces $(\delta \mathrm{p})$, and hydrogen bonding forces $(\delta \mathrm{h})$ to the overall cohesive forces $(\delta \mathrm{t})$ that hold the polymers together. They are based on the molar attraction values of their individual molecular components and can be calculated according to the method of Hoy using commercially available software.

The application of these parameters demonstrated a Hoy's $\delta$ h value of $14.8(\mathrm{~J} / \mathrm{cm} 3)^{1 / 2}$ in collapsed collagen. As a consequence, the use of dental adhesives components which $\delta$ h values are above $14.8(\mathrm{~J} / \mathrm{cm} 3)^{1 / 2}$, can promote the expansion of the collagen by breaking interpeptide hydrogen-bonding. Such evidence suggests the use of monomer solvents, as ethanol $(\delta \mathrm{h}=20.0)$ and water $(\delta \mathrm{h}=40.4)$ to break the inter-peptide hydrogen bonds of the collagen and to soften and expand it rapidly [56]. These solvents may be blended with 2-hydroxyethyl methacrylate (HEMA), which is an excellent, relatively hydrophilic, nonvolatile, polymerizable solvent for dimethacrylates [22].

The use of computer modeling analysis makes it possible to investigate at the atomic-level biochemical interactions that occur in localized sites of large target molecules (i.e. collagen), due to the presence of other reactive molecules (i.e. adhesive monomers) in the vicinity [57]. The computer simulations can mimic rapid energy 
fluctuations between different stable/metastable states reflecting conformational changes of the adhesive molecules. The results of the study show the effect of molecular species (ligand), such as liquid primer molecules, which may lead to multiple binding profiles in a complex target structure such as collagen. Data show no evidence of any primary bonds between collagen and the monomer molecules. The interaction is therefore primarily due to secondary bonds including van der Waals interactions together with limited electrostatic and hydrogen-bonding interactions. Interactions may occur when specific features (functional groups and spacer features) in ligands in low-energy (stable) conformational states are configured in alignment with complementary features in the collagen target. Thus charge, hydrogen bonding, and spatial alignment between the target and the ligand may result in electrostatic, hydrogen bonding, and steric complementarity, whose different distributions may influence the hybrid layer bond strength.

\section{-Self etching Adhesives}

Self-etching bonding systems were introduced to resolve some of the problems which have been experienced when using etch-andrinse adhesives.

Most self-etching adhesives contain specific functional monomers that, to a large extent, determine the adhesive performance. Functional monomers are used with the intent of etching tooth substrates, enhancing monomer penetration and also (potentially) establishing a chemical interaction between the adhesive and the dental substrates $[15,16]$.

With self etching adhesives the demineralization and resin infiltration procedures are ideally performed almost simultaneously and to the same depth, due to the presence of the acidic functional monomers that demineralize and infiltrate the tooth surface at the same time. Smear layer and the mineral phase are not removed from the surfaces but acidic monomers penetrate into the tissue through the porosities of the smear and the superficial layer of the dentine. This facilitates the interaction of the monomers with both the hydroxyapatite (HAp) mineral phase as well as the collagen phase of the tissue [55].

So far, commonly used functional monomers in commercial self etching adhesives are phosphate monomers, such as 10methacryloxydecyl dihydrogen phosphate (MDP) and 2-methacryloxyethyl phenyl hydrogen phosphate (Phenyl-P). Both monomers have been used as etching monomers in self-etching primers and in bonding agents to promote resin diffusion and adhesion [58].

Another consequence of the simultaneous demineralization/infiltration procedure of the self etching adhesives is the reduction of clinical application time compared to the etch-and-rinse adhesives, as well as the significant decrease of post-operative sensitivity due to the incorporation of the smear layer and smear plugs into the hybrid layer.

Self etching systems are classified as two-step and one step self etching regardless of the actual numbers of steps involved in the bonding: 1) Two-step self-etching adhesives are based on the separate application of a hydrophilic self-etching primer followed by a hydrophobic bonding resin 2) "one-step" adhesives, also referred as "all-in-one" combine the self-etching primer and the bonding resin into a single step. However, such "one-step" adhesives may require several applications of the adhesive and thus in reality more steps are necessary for good clinical results.

\section{- Two-step self-etching adhesives}

The primer and bonding resin formulations contain a mixture of resin monomers, and light, chemical, or dual cure initiators and other additives. Water as an ionizing medium is also contained in all the self etching primers in order to enable the etching process [59]. The primer includes hydrophilic functional monomers whose acidity allows for the demineralization process of the self etching adhesives. Additionally, they may interact with HAp and collagen matrix phases with a series of atomic-level interactions, which may play a critical role in the overall adhesion process.

Aggressiveness depends on the $\mathrm{pH}$ of the self etching adhesive that range around $\mathrm{pH} 1$ or below in the case of strong self etching, to $\mathrm{pH} 2.0$ in mild self etching adhesives. With the increase in depth, the acidic monomers are gradually buffered by the mineral content of the dental tissue thus losing their ability of etching [60].

Primers with a $\mathrm{pH}$ around 1 (strong self-etching adhesives) cause a rather deep demineralization. On the enamel, the etching pattern is similar to that obtained after a phosphoric-acid treatment following an etch-and rinse-approach (Fig. 5) [61,62]. On the dentine, collagen is exposed and nearly all hydroxyapatite is dissolved [63]. Consequently, morphological characteristics of "strong" selfetching systems are very similar to those produced by etch-andrinse adhesives. The dentine hybridization is primarily diffusionbased and abundant formation of resin tags may be possible. However, these systems showed a gradual decrease of the bond strength, particularly when oral stress was simulated $[64,65]$.

Mild self etching systems have a $\mathrm{pH}$ of around 2. On the enamel the pattern of etching with mild self etching systems is minimal (Fig. 5), resulting in shallow inter-crystallite infiltration of the resin and lack of inter-prismatic resin tag formation $[66,67]$.

On the dentine mild self etching systems are able to partially demineralize and penetrate the dentinal surface up to $1 \mu \mathrm{m}$ depth, creating hybrid layers that are thinner and with less pronounced resin tag formation than strong self etching and etch-and-rinse adhesives [63] (Fig. 6). However, with mild self etching very high dentine bond strength data have been reported. These are similar to those obtained with etch-and-rinse adhesives $[68,69]$ and their stability under stress is higher compared to the strong self-eching adhesives [64].

The so called 'AD-concept' or 'Adhesion- Decalcification concept' [15] explains the different behaviors of the two step self etching adhesives. Carboxylic acid-based monomers like 4-MET (4methacryloxyethyl trimellitic acid), phosphate-based monomers, like as Phenyl-P, and MDP which are part of the acidic primer, have a chemical bonding potential with calcium of residual hydroxyapatite, as demonstrated by the 'AD-concept'. According to this concept all the acids interact with the calcium of hydroxyapatite forming ionic bonds. However, the stability of the formed salts depends on the proper mode of interaction of the monomers and it is inversely related to the monomer acidity. Thus, Phenyl-P and 4META, like for phosphoric acid molecules, strongly interact with enamel and dentine tissues forming ionic bonds with hydroxyapatite calcium. However, due to instability of the calcium phosphate/carboxylate compound, it undergoes degradation and finally de-bonding. The result is that calcium is deeply removed from the enamel/dentine surface leading to the typical pattern of enamel etching and a relatively deep demineralization of the dentine. The latter favors hybrid layers of about 3-5 $\mu \mathrm{m}$ which are totally calcium-deprived. On the contrary, the interaction of MDP on both enamel and dentine gives rise to a superficial demineralization of the surfaces with residual HAp within the hybrid layer and more stables salts.

Thus, MDP is considered as one of the most promising monomers for chemical bonding to dental substrates, as it establishes an ionic bond with HAp most readily and intensively, even in the short exposure time of 30 seconds. In contrast, Phenyl-P has very limited chemical bonding capacity, with formation of weak ionic binding with HAp and highly soluble reaction salts. The above considerations were demonstrated by X-ray photoemission spectroscopy and TEM studies [15] showing the capacity of MDP to form a regularly layered structure on the HAP surface, within which calcium salts are deposited [70] (Figs. 7, 8). The stronger interaction energy of 


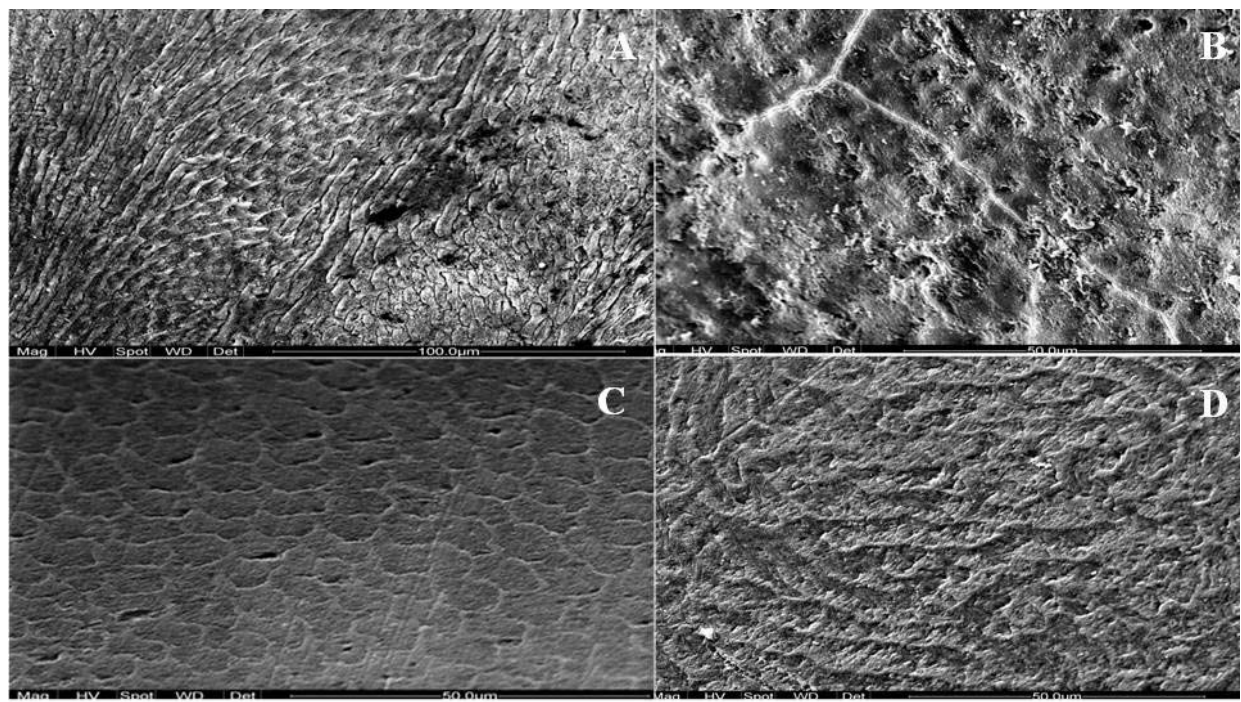

Fig. (5). Enamel etching pattern of different self etching systems. In (A) Resulcin AquaPrime (Merz Dental, Lütjenburg, Germany) a 2-methacryloxyethyl phenyl hydrogen phosphate (phenyl-P), a $0.5 \mathrm{pH}$ acidic primer whose enamel etching pattern is similar to that obtained after a phosphoric-acid treatment. In (B) Artegal One, (Merz Dental GmbH, Eetzweg, Lütjenburg) a modified methacrylates, phosphoric acid esters primer, pH 1.3, which shows a geometrically etching of enamel with shallow crater-like depressions and less visible boundary wall appearance. In (C) Clearfil Protect Bond (Kuraray, Osaka, Japan) a 10methacryloyloxydecyl dihydrogen phosphate (MDP) primer, $\mathrm{pH}$ 2.8; and in (D) Cleafil S3 Bond (Kuraray, Osaka, Japan) a 10-methacryloyloxydecyl dihydrogen phosphate (MDP), Bis-phenol A diglycidylmethacrylate (Bis-GMA), 2-hydroxyethyl methacrylate (HEMA) "one step" self etching system, pH 2.7. Both the mild MDP primers of Clearfil Protect Bond and Clearfil S3 give rise to a regular pattern of enamel etching with micro-porous aspect of the inter and intraprismatic surfaces.

Courtesy of Dott. Giorgio Castelli, University of Sassari, Italy.

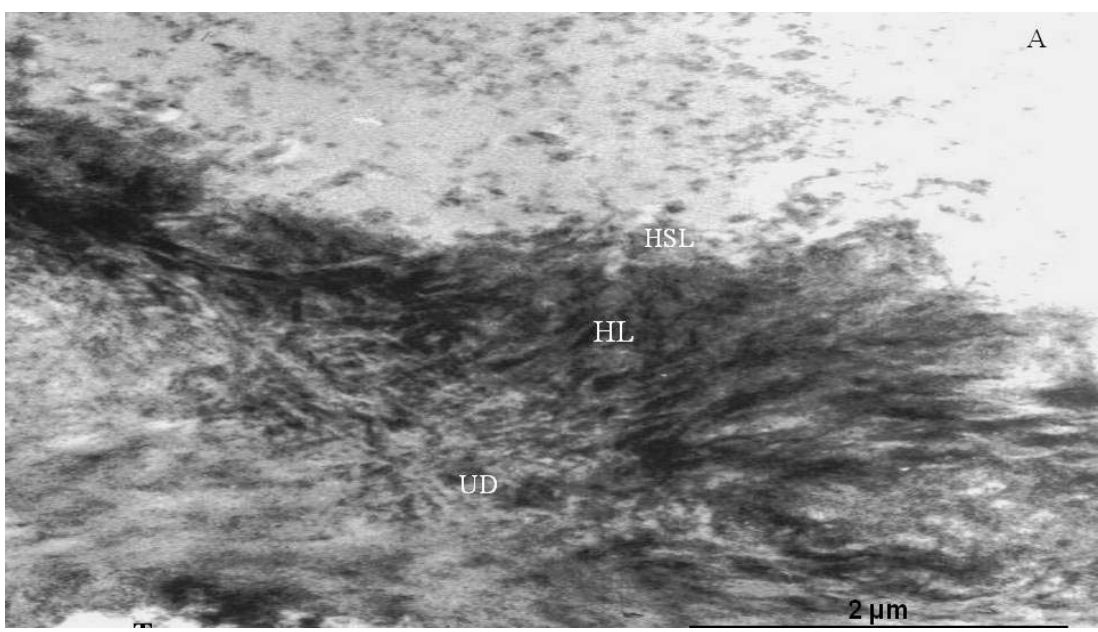

Fig. (6). Demineralized ultrathin section of the resin-dentine interdiffusion produced by Clearfil SE Bond (Kurary, Osaka, Jappan) a mild self etching system based on the same 10-MDP technology of Kuraray adhesives. In the first step of priming, the mild acidic MDP primer allows for partial demineralization of the fibrils with exposure of the collagen HAp. Additionally, the MDP- primer interacts with HAp and collagen matrix phases with a series of atomic-level interactions, which may play a critical role in the overall adhesion process. The smear layer is usually retained and anchored to the bond. The second step concerns the application of the hydrophobic adhesive resin which completes the formation of the hybrid layer.

$\mathrm{HL}=$ hybrid layer; $\mathrm{HSL}=$ hybridized smear layer; $\mathrm{T}=$ tubule; $\mathrm{A}=$ adhesive; $\mathrm{UD}=$ unaltered dentine

MDP with HAP in comparison to PhenylP was also evaluated using computed modeled and AutoDock program of analysis [54].

As a consequence of its specific interaction with dentin, MDP containing self etching adhesives may help inhibit secondary caries. An electron dense zone was observed under the hybrid layer formed by MDP containing self etching adhesives after exposure to an artificial demineralizing solution $(\mathrm{pH} 4.5)$ for 90 mins and then $5 \%$ sodium hypochlorite for 20 mins. This area was identified as an "acid-base resistant zone" (ABRZ) [71]. This ABRZ contains densely packed crystallites probably formed by resin-infiltrated dentine. This suggests that some chemical reaction of HAp and MDP may take place in this zone, enhancing resistance against demineralization from acid attack [72]. Although the mechanism of formation of the acid-base resistant zone is unclear, these new findings suggested that dentine adjacent to the adhesive-dentine interface is different from the normal dentine, which shows the potential to resist an acid attack from microorganisms in primary and secondary caries. Therefore these studies suggest a reinforcement of the dentine by self etching adhesive technology. Formation of this reinforced dentine with superior qualities than the normal dentine may 


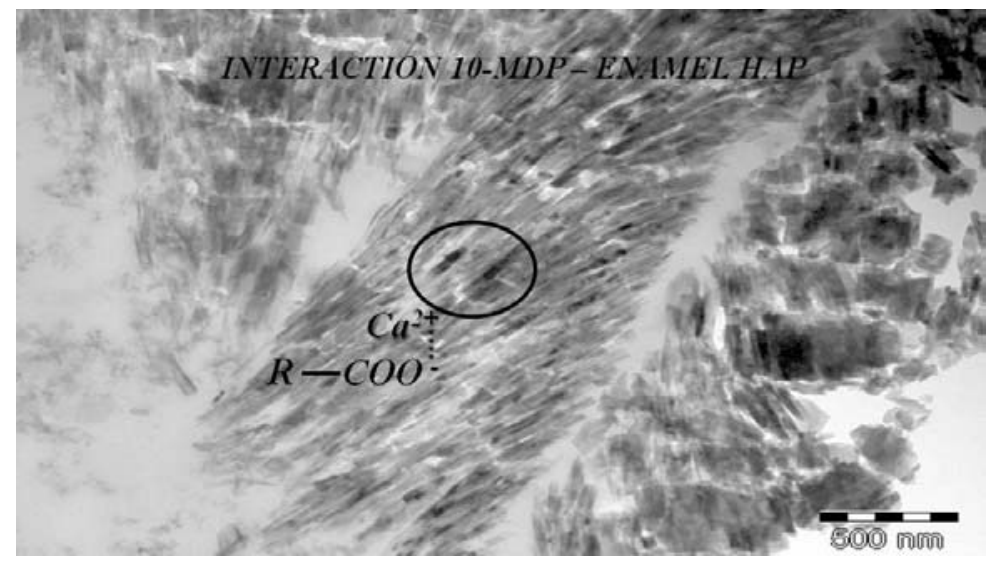

Fig. (7). Non-decalcified ultrathin section of resin-enamel interdiffusion produced by the use of Clearfil Protect Bond, "two step" self etching system, pH 2.8 . The fugure shows a three-dimensional nano-retentive appearance on the enamel surface with inter and intra-crystallite hybridization. Several macro-tags and micro-tags of crystal enveloped by the resin can be seen at high magnification.

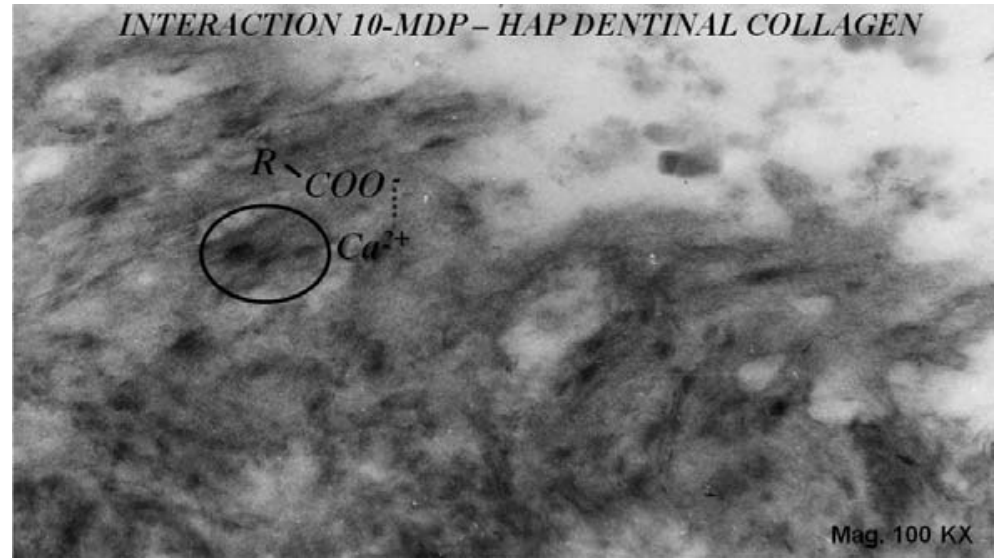

Fig. (8). TEM demineralized section of the dentine interdiffusion of Clearfil SE Bond. The functional monomer MDP allows for a strong ionic bond with the calcium exposed in Hap. Its interaction energy is dominated by electrostatic interaction energy due to the ionic character of the bond and the close distance of approach of the anionic phosphate functionality of MDP to the cationic calcium lattice sites on HAp crystal.

become a key strategy in the future. Further studies should be carried out to elucidate the mechanism of this modified dentine layer.

\section{- One-Step Self Etching Adhesives}

One-Step Self Etching Adhesives are complex mixtures of hydrophilic and hydrophobic components which produce thinner hybrid layers compared to two step adhesives and etch-and-rinse adhesives. A greater simplification of the bonding procedure in onestep may have clinical advantages, but some studies on their bonding performance indicated that these thin hybrid layers were prone to be less polymerised [73] and permeable [53,54,74,75,76]. They are also very hydrophilic and absorb water from dentinal tubules by osmosis. Unreacted monomers or oligomers can leach out from the polymer during water sorption with subsequent polymer expansion. Generally, increases in water sorption are associated with increases in solubility, hydrolytic degradation products, nanoleakages and a resultant decline in bond strength [77].

These problems may be due to the high concentrations of hydrophilic monomers, such as HEMA, and to the need for a more hydrophobic coupling resin layer [53]. HEMA is added in high concentration by manufacturers in order to facilitate Bis-GMA solubility, contained in the same mixture, to produce a tough, highly cross-linked polymer network. However, after polymeriza- tion, poly (HEMA) attracts water [78] causing water sorption that is proportional to the HEMA concentration.

The movement of fluids by water attraction results in droplets or voids which can be visualized in the SEM. This is associated with the presence of micro-phase separation of hydrophilic and hydrophobic components within these permeable membranes (Fig. 9).

Moreover, an increase in the permeation of water to the hydrophilic adhesive layer of the one-step adhesives has been related to the hydration of the underlying dentine [53]. A highly significant correlation has been observed between the permeability of these adhesives and the number of fluid droplets on the resin/dentine surface with the amount of water supplied by the wet surface of the deep dentine under positive pressure [78]. Conversely, the absence of fluid droplets was documented when one-step adhesives were applied to a caries-affected dentine in which occluded dentinal tubules blocked the intratubular permeation of fluids [79].

Calculation of the Hoy's solubility parameters can provide important predictions of water sorption and solubility in one step selfetching adhesives. Polymers lacking polar groups or constituents incapable of forming hydrogen bonds are relatively hydrophobic and have high $\delta \mathrm{d}$ values relative to their $\delta \mathrm{p}$ and $\delta \mathrm{h}$ values. Polymers containing polar groups that cannot form ionic attractions or hydro- 


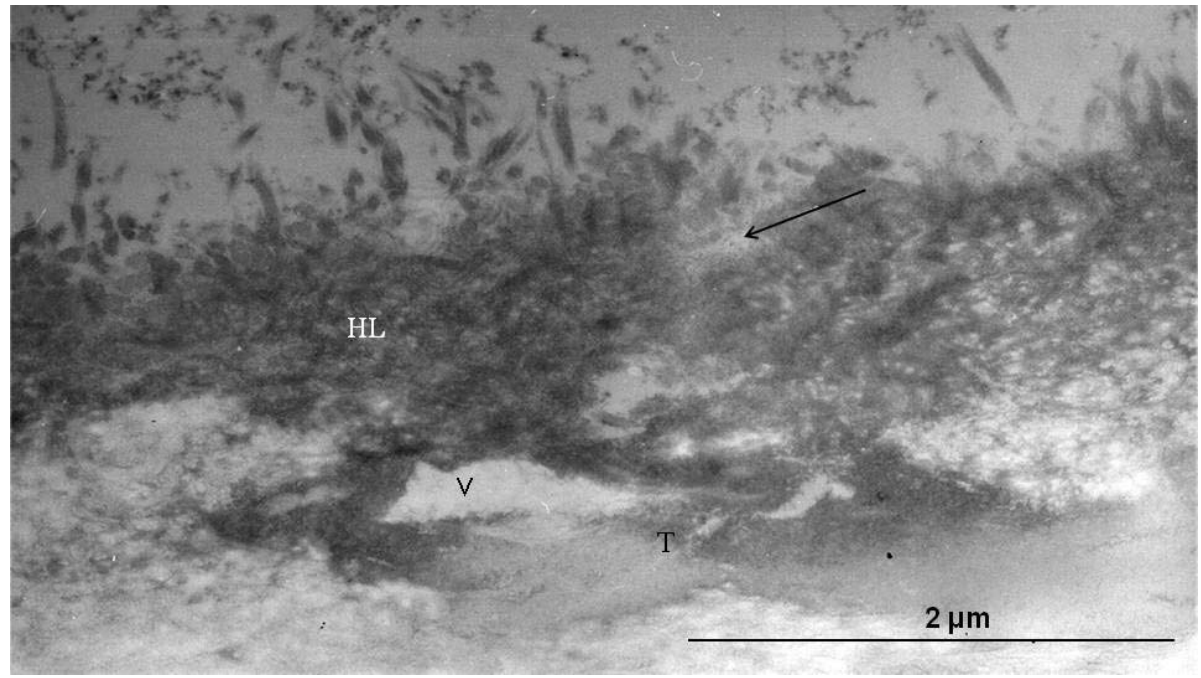

Fig. (9). TEM demineralized section of the very thin hybrid layer formed by Clearfil S3 Bond "one step" self etching system. The interdiffusion was done in deep dentine in in vivo conditions and samples were extracted after $10 \mathrm{~min}$ of pulpal pressure. It is notable the high hydrophilic aspect of the hybrid layer which shows voids $(\mathrm{V})$ in the tubule and channel of water (arrow) toward the interface by means of the unsealed tubules. These common problems in the one step self etching systems are connected to the high concentrations of hydrophilic monomers, such as 2-hydroxyethyl methacrylate (HEMA) and solvents in the formulation, and to the need for a more hydrophobic coupling resin layer

gen bonds (i.e. halogen-substituted polymers) are moderately hydrophilic and have relatively high $\delta p$ values. Polymers containing hydroxyl groups, amines, carboxylic acids or phosphates are very hydrophilic and have high $\delta \mathrm{p}$ and $\delta \mathrm{h}$ values.

To optimize the composition of self-etching adhesives, just enough HEMA should be added to wet the dentine and prevent phase separations of dimethacrylates, without causing excessive water sorption/solubility. However, such compromises may not create optimal bonds [56]

Thus, acrylamide-based adhesive systems have been designed to overcome the problem of hydrolytic instability of one-step self etching adhesives. Acrylamides are introduced as a cross-linking mechanism in hydrophilic resin formulations $[36,80]$ promising a better performance of the latest generation of one-step adhesives.

\section{CONCLUSIONS}

Dental adhesives are used for a wide range of clinical applications. They are available as etch-and-rinse systems and self-etch systems. Both systems result in etching of the tooth surface, and micromechanical bonding of enamel and dentine following the polymerisation. A chemical bond to dentin is discussed for one monomer, MDP.

Etch-and-rinse adhesive systems generally perform better on enamel than self-etching systems which may be more suitable for the bonding to dentine. Because of the less retentive etching pattern in enamel after using mild self-etching adhesives marginal discoloration of the restorations may occur with such adhesives. This is a special clinical problem in anterior teeth. Therefore, etch-and-rinse approaches may be advantageous the anterior region. Self-etching adhesives (especially mild preparations) may have advantages in the posterior region: here the cavity surface is mainly dentin and these adhesive show excellent adhesion to dentin.

Careful consideration of several factors is essential in selecting the bonding procedure and adhesive system for the vital dentine, in order to combine longevity of the restoration, lack of secondary caries and of pulp damage not only due to eluted monomers but also due to bacteria and their products penetrating developing gaps between material and cavity walls.

\section{CONFLICT OF INTEREST}

The authors confirm that this article content has no conflicts of interest.

\section{ACKNOWLEGMENTS}

E.M. and E.C. equally contributed to this paper.

\section{REFERENCES}

[1] Nicholson JW, Chemistry of glass-ionomer cements: a review. Biomaterials 1998; 19: 485-94

[2] Walls AWG, Glass Polyalkenoate (glass-ionomer) cements: a rewiew. J Dent 1986; 14: 231-46

[3] Wilson AD, Developments in glass-ionomer cements 1989; 5: 43866

[4] Xie D, Brantley WA, Culbertson BM, Wang G, Mechanical properties and microstructures of glass-ionomer cements. Dent Mat 2000; 2: 129-38

[5] Cramer NB, Stansbury JW , Bowman CN, Recent advances and developments in composite dental restorative materials. J Dent Res, 2011; 4: 402-16

[6] Atai M, Pahlavan A, Moin N. Nano-porous thermally sintered nano silica as novel fillers for dental composites. Dent Mater 2012 (2): 133-45.

[7] Hosoya Y, Shiraishi T, Odatsu T, et al. Effects of polishing on surface roughness, gloss, and color of resin composites. J Oral Sci 2011; (3): 283-91

[8] Hosseinalipour M, Javadpour J, Rezaie H, Dadras T, Hayati AN Investigation of mechanical properties of experimental BisGMA/TEGDMA dental composite resins containing various mass fractions of silica nanoparticles. J Prosthodont 2010 (2): 112-7.

[9] Torres CR, Borges AB, Goncalves SE, Pucci CR, de Araujo MA, Barcellos DC. Clinical evaluation of two packable resin-based composite restorations: a three-year report. Gen Dent 2010; (4): 338-43.

[10] Majety KK, Pujar M. In vitro evaluation of microleakage of class II packable composite resin restorations using flowable composite and resin modified glass ionomers as intermediate layers. J Conserv Dent 2011; (4): 414-7

[11] Buonocore MG. A simple method of increasing the adhesion of acrylic filling materials to enamel surfaces. J Dent Res 1955; 34: 849-53. 
[12] Nakabayashi N, Kojima K, Masuhara E. The promotion of adhesion by the infiltration of monomers into tooth substrates. J Biomed Mater Res 1982; 16: 265-73.

[13] Nakabayashi N, Nakamura M, Yasuda N. Hybrid layer as a dentinbonding mechanism. J Esthet Dent 1991; 3: 133-8.

[14] Yoshida Y, Van Meerbeek B, Nakayama Y, et al. Evidence of chemical bonding at biomaterial-hard tissue interfaces. J Dent Res 2000; 79: 709-14.

[15] Yoshida Y, Nagakane K, Fukuda R, et al. Comparative study on adhesive performance of adhesive monomers. J Dent Res 2004; 83: 454-8.

[16] Bertassoni LE, Stankoska K, Swain MV. Insights into the structure and composition of the peritubular dentin organic matrix and the lamina limitans. Micron 2012; 43: 229-36.

[17] Pashley DH, Horner JA, Brewer PD. Interactions of conditioners on the dentin surface. Oper Dent 1992; 17: 137-50.

[18] Burrow MF, Takakura H, Nakajima M, Inai N, Tagami J, Takatsu $\mathrm{T}$. The influence of age and depth of dentin on bonding. Dent Mater 1994; 10: 241-6.

[19] Perdigao J, Swift EJ, Denehy GE, Wefel JS, Donly KJ. In vitro bond strengths and SEM evaluation of dentin bonding systems to different dentin substrates. J Dent Res 1994; 73: 44-55.

[20] Nakajima M, Sano H, Burrow MF, et al. Tensile bond strength and SEM evaluation of caries-affected dentin using dentin adhesives. J Dent Res 1995; 74: 1679-88.

[21] Demirci M, Hiller KA, Bosl C, Galler K, Schmalz G, Schweikl H. The induction of oxidative stress, cytotoxicity, and genotoxicity by dental adhesives. Dent Mater 2008; 24: 362-71

[22] Van Landuyt KL, Snauwaert J, De Munck J, et al. Systematic review of the chemical composition of contemporary dental adhesives. Biomat 2007; 28: 3757-85.

[23] Schmalz G. Concepts in biocompatibility testing of dental restorative materials. Clin Oral Invest 1997; 1: 154-62.

[24] Wej Y, Silikas N, Zhang Z, Watts DC. Diffusion and concurrent solubility of self-adhering and new resin-matrix composites during water sorption/desorption cycles. Dent Mat 2011; 27: 197-205.

[25] Ferracane JL. Hygroscopic and hydrolytic effects in dental polymer networks. Dent Mater 2006; 22: 211-22

[26] Watanabe I, Nakabayashi N, Pashley DH. Bonding to ground dentin by a phenyl-P self-etching primer. J Dent Res 1994; 73: 121220.

[27] Moszner N, Salz U, Zimmermann J. Chemical aspects of self etching enamel-dentin adhesives: a systematic review. Dent Mater 2005; 21: 895-910.

[28] Imazato S, Russell RR, McCabe JF. Antibacterial activity of MDPB polymer incorporated in dental resin. J Dent 1995; 23: 17781.

[29] Milia E, Campus G, Santini A. Resin adhesion to sclerotic dentin: an in vivo TEM study of a new antibacterial system. J Dent Res 82 (Special Iss B) 2003; 255. Abstract \#1954.

[30] Valle D, Solinas G, Santini A, Gallottini L, Milia E. Antibacterial activity of a new self-etching adhesive system: in vivo TEM observation. International Kuraray Symposium of Adhesive Dentistry, Dusseldorf, Germany 2004, Abstract \#11.

[31] Giammanco GM, Cumbo EM, Luciani A, Gallina G, Mammina C, Pizzo G. In vitro evaluation of the antibacterial activity of cured dentin/enamel adhesive incorporating the antimicrobial agent MDPB. New Microbiol 2009; 32: 385-90

[32] Milia E, Cotti E, Sotgiu G, Masarin M, Manunta A, Gallina G. In vivo, morphological and clinical effect of a desensitizing agent. Dent Mat 2010; Supplement 26: 1 e67.

[33] Ferracane JL, Greener EH. The effect of resin formulation on the degree of conversion and mechanical properties of dental restorative resins. J Biomed Mater Res 1986; 20: 121-31.

[34] Ferracane JL. Hygroscopic and hydrolytic effects in dental polymer networks. Dent Mat 2006; 22: 211-22.

[35] Tay FR, Pashley DH, Suh BI, Carvalho RM, Itthagarun A. Singlestep adhesives are permeable membranes. J Dent 2002; 30: 371-82, a.

[36] Friedman M. Chemistry, biochemistry, and safety of acrylamide. A review. J Agric Food Chem 2003; 51: 4504-26.

[37] Spagnuolo G, Galler K, Schmalz G, Cosentino C, Rengo S, Schweikl H. Inhibition of phosphatidylinositol 3-kinase amplifies TEGDMA-induced apoptosis in primary human pulp cells. J Dent Res 2004; 83: 703-7.
[38] Schweikl H, Spagnuolo G, Schmalz G. Genetic and cellular toxicology of dental resin monomers. J Dent Res 2006; 85: 870-7.

[39] Vajrabhaya L, Korsuwannawong S, Bosl C, Schmalz G. The cytotoxicity of self-etching primer bonding agents in vitro. Oral Surg Oral Med Oral Pathol Oral Radiol Endod 2009; 107: 86-90.

[40] Van Meerbeek B, De Munck J, Yoshida Y, et al. Buonocore memorial lecture. Adhesion to enamel and dentin: current status and future challenges. Oper Dent 2003; 28: 215-35

[41] Erickson RL, Barkmeier WW, Latta MA. The role of etching in bonding to enamel: A comparison of self-etching and etch-andrinse adhesive systems. Dental Mater 2009; 25: 1459-67

[42] Van Meerbeek B, Vargas S, Inoue S, et al. Adhesives and cements to promote preservation dentistry Oper Dent 2001; Supplement 6 : 119-14.

[43] Kanca JA. Improving bond strength through acid etching of dentin and bonding to wet dentin surfaces. J Am Dent Assoc 1992; 123 $35-43$.

[44] Kanca JA. Wet bonding: The effect of drying time and distance. Am J Dent 1996; 9: 273-76.

[45] Maciel KT, Carvalho RM, Ringle RD, Preston CD, Russell CM, Pashley DH. The effects of acetone, ethanol, HEMA, and air on the stiffness of human decalcified dentin matrix. J Dent Res1996; 75: 1851-8.

[46] Milia E, Lallai MR, Garcia-Godoy F. In vivo effect of a self etching primer on dentin. Am J Dent 1999; 4: 167-71.

[47] Jacobsen T, Söderholm KJ. Some effects of water on dentin bonding. Dent Mater 1995; 11: 132-6.

[48] Yoshiyama M, Carvalho RM, Sano H, Homer JA, Brewer PD, Pashley DH. Comparison of interfacial morphology and strength of bonds made to superficial and deep dentin. Am J Dent 1996; 8: 297-302.

[49] Pashley DH, Carvalho RM. Dentine permeability and dentine adhesion J Dent 1997; 5: 355-72

[50] Sano H, Takatsu T, Ciucchi B, Horner JA, Matthews WG, Pashley DH. Nanoleakage: Leakage within the hybrid layer. Oper Dent 1995; 20: 18-25.

[51] Pioch T, Staehle HJ, Duschner H, Garcia-Godoy F. Nanoleakage at the composite-dentin interface: A review. Am J Dent 2001; 14: 252-58.

[52] Tay FR, Pashley DH, Yoshiyama M. Two modes of nanoleakage expression in single-step adhesives. J Dent Res 2002; 81: 472-76, b.

[53] Tay FR, Pashley DH. Water treeing-A potential mechanism for degradation of dentin adhesives. Am J Dent 2003; 16: 6-12.

[54] Vaidyanathan TK, Vaidyanathan J: Recent Advances in the Theory and Mechanism of Adhesive. Resin Bonding to Dentin: A Critical Review. J Biomed Mater Res Part B: Appl Biomater 2009; 88B: 558-78.

[55] Pashley DH, Carvalho RM, Tay FR, Agee KA, Lee KW. Solvation of dried dentin matrix by water and other polar solvents. Am J Dent 2002; 15: 97-102

[56] Barton AFM. Handbook of solubility parameters and other cohesion parameters. CRC Press: Boca Raton, 1991; 69-156.

[57] Vaidyanathan TK, Vaidyanathan J, Kerrigan J. Evaluation of biochemical interactions of self-etch dentin adhesive primer molecules with type 1 collagen: Computer modeling and in vitro binding analysis. Acta Biomaterialia 2007; 3: 705-14

[58] Van Meerbeek B, Yoshihara K, Yoshida Y, Mine A, De Munck J, Van Landuyt KL. State of the art of self-etch adhesives. Dent Mater 2011; 27: 17-28.

[59] Salz U, Mucke A, Zimmermann J, Tay FR, Pashley DH. pKa value and buffering capacity of acidic monomers commonly used in selfetching primers. J Adhes Dent 2006; 8: 143-150

[60] Castelli G, Gallottini L, Sotgiu G, Gallina G, Lumbau A, Milia E. Evaluation of the enamel etching pattern of self-etching adhesives. Dent Mater 2010; 26 Supplement 1, e4.

[61] Pashley DH, Tay FR Aggressiveness of contemporary self-etching adhesives. Part II: Etching effects on unground enamel Dent Mat 2001; 17: 430-44.

[62] Milia E, Santini A. Ultrastructural transmission electron microscopy (TEM) study of hybrid layers formed beneath a one-bottle adhesive system using the total-etch technique and a self-etching system. Quintessence Int 2003; 34: 447-52.

[63] Inoue S, Koshiro K, Yoshida Y, De Munck J et al. Hydrolytic stability of self-etch adhesives bonded to dentin. J Dent Res 2005 84: 1160-4. 
[64] Salz U, Zimmermann J, Zeuner F, Moszner N. Hydrolytic stability of self-etching adhesive systems. J Adhes Dentistry 2005; 7: 10716.

[65] Hannig M, Bock H, Bott B, Hoth-Hannig W. Inter-crystallite nanoretention of self-etching adhesives at enamel imaged by transmission electron microscopy. Eur J Oral Sci 2002; 11: 464-70.

[66] Perdigão J, Geraldeli S. Bonding characteristics of self-etching adhesives to intact versus prepared enamel. J Esthet Restor Dent 2003; 15: 32-42.

[67] Sattabanasuk V, Shimada Y, Tagami J. The bond of resin to different dentin surface characteristics Oper Dent 2004; 29: 333-41.

[68] Senawongse P, Harnirattisai C, Shimada Y, Tagami J. Effective bond strength of current adhesive systems on deciduous and permanent dentin Oper Dent 2004; 29 196-202.

[69] Yoshihara K, Yoshida Y, Nagaoka N, et al. Nano-controlled molecular interaction at adhesive interfaces for hard tissue reconstruction. Acta Biomater 2010; 6: 3573-82.

[70] Waidyasekera K, Nikaido T,. Weerasinghe DS, Ichinose S, Tagami J. Reinforcement of dentin in self-etch adhesive technology: A new concept J Dent 2009; 37: 604-9.

[71] Inoue G, Nikaido T, Foxton RM, Tagami J. The acid-base resistant zone in three dentin bonding systems. Dent Mater J 2009; 28: 71721.

[72] Van Landuyt K, Cardoso M, De Munck J, et al. Optimization of the concentration of photo-iniziator in a one step self-etch adhesive. Dent Mater 2009; 25: 982-8.

[73] Itthagarun A, Tay FR, Pashley DH, Wefel JS, Garcia-Godoy F, Wei SHY. Single-step, self-etch adhesives behave as permeable membranes after polymerization. Part III. Evidence from fluid conductance and artificial caries inhibition. Am J Dent 2004; 17: 394 400 .

[74] Wang Y, Spencer P. Continuing etching of an all-in-one adhesive in wet dentin tubules. J Dent Res 2005; 84: 350-4.

[75] Yoshida E, Uno S, Nodasaka Y, Kaga M, Hirano S. Relationship between water status in dentin and interfacial morphology in all-inone adhesive. Dent Mater 2007; 23: 556-60.

[76] Hashimoto M, Fujita S, Kaga M, Yawaka Y. Effect of water on bonding of one-bottle self-etching adhesives. Dent Mater J 2008; 27: $172-8$.

[77] Ito S, Hashimoto M, Wadgaonkar B, et al. Effects of resin hydrophilicity on water sorption and changes in modulus of elasticity. Biomat 2005; 26: 6449-59.

[78] Sauro S, Mannocci F, Toledano M, Osorio R, Thompsonand I, Watson TF. Influence of the hydrostatic pulpal pressure on droplets formation in current etch-and-rinse and self-etch adhesives: A video rate/TSM microscopy and fluid filtration study. Dent Mater 2009; 25: 1392-402

[79] Tay FR, Pashley DH, Hiraishi N, et al. Tubular occlusion prevents water-treeing and though- and through fluid movement in a singlebottle, one-step self-etch adhesive model. J Dent Res 2005; 84 891-6.

[80] Santini Ario, Milia Egle, Miletic Vesna: A review of SEM and TEM studies on the hybridisation of dentine. In Microscopy: Science, Technology, Applications and Education. $4^{\text {rd }}$ Ed. MéndezVilas \& Díaz: Badajoz 2010; Vol 1, pp. 256-67. 